\title{
Contemplations on Dirac's equation in quaternionic coordinates
}

\author{
Dirk Schuricht and Martin Greiter
}

\author{
Institut für Theorie der Kondensierten Materie, \\ Universität Karlsruhe, Postfach 6980, D-76128 Karlsruhe
}

August 17, 2004

\begin{abstract}
A formulation of Dirac's equation using complex-quaternionic coordinates appears to yield an enormous gain in formal elegance, as there is no longer any need to invoke Dirac matrices. This formulation, however, entails several peculiarities, which we investigate and attempt to interpret.
\end{abstract}

\section{Introduction}

One of the breakthroughs in the development of quantum mechanics was the discovery of a linear, relativistic wave equation for fermions by Dirac in 1928, which is known as Dirac's equation [1, 2]. This equation identified the electron spin as an intrinsic quantum number and predicted the existence of antiparticles. The corresponding Lagrangian is at the basis of quantum electrodynamics, and hence has played a major role on our path towards an understanding of elementary particles and their interactions. Dirac derived the equation, in a sense, by "taking the square root" of the Klein-Gordon equation, which is quadratic in time and space derivatives and the minimal version of a relativistically covariant wave equation. In order to overcome the technical obstacles in writing a linear equation, Dirac proposed that the 
wave equation should be considered as a matrix equation for complex spinorvalued wave functions rather than complex scalar functions. The equation contains a set of $4 \times 4$ matrices, the so-called Dirac matrices, for which different representations satisfying the so-called Dirac algebra are possible.

To the student who learns about these matrices for the first time, however, they may look somewhat arbitrary. One can easily derive the algebra and choose a specific representation, but nonetheless, on purely aesthetic grounds these matrices remain peculiar. A rather provocative statement by Einstein comes to mind: "When judging a physical theory, I ask myself whether I would have made the Universe in that way had I been God". In the context of Dirac's equation, there is little sense in even questioning the correctness of the theory, but one may still ask whether there might not be a way to formulate it without availing oneself of these peculiar matrices.

The structure of the matrices in Dirac's equation is intimately linked to relativistic covariance. Special relativity, however, can not only be formulated using the standard Lorentz four-vector notation, but also using a complexquaternionic (CQ) parametrisation of space-time, as we have emphasised in a pedagogical review recently [3]. This parametrisation yields a significant gain in formal elegance, as Lorentz transformations are no longer implemented by multiplication with $4 \times 4$ matrices, but by multiplication with CQ numbers. Maxwell's four equations reduce to a single CQ equation.

The question we wish to address in this article is whether one can formulate Dirac's equation (and quantum electrodynamics) in CQ coordinates, and to which extent such a formulation is equivalent to, or can be brought into a form equivalent to the standard theory. We will find that relativistic covariance implies immediately that the entities in the now two-component Dirac spinors are no longer complex numbers, but CQ numbers, and hence somewhat reminiscent of quaternionic quantum mechanics 4. Among the peculiarities intrinsic to this formulation is an apparent doubling of solutions [5, 6, 7, which we attribute to the existence of an additional, global $S U(2)$ gauge symmetry, as well as the possibility to write solutions which carry spin one-half but do not possess a direction for the quantisation of this spin, which we eliminate from the spectrum of physical states through a suitable condition.

Finally, we conclude that while it is indeed possible to gain an intriguing amount of elegance by formulating Dirac's equation in CQ coordinates, we have either failed to recognise a deeper physical principle at work or the price one has to pay for this gain in elegance is significant. 


\section{Special relativity}

We begin with a review of quaternions and the formulation of special relativity within the framework of CQ numbers [3]. We introduce a complex algebra with generators $1, @ \in \mathbb{C}$, such that

$$
\varliminf^{2}=-1
$$

as well as a quaternionic algebra with generators $1, i, j, k \in \mathbb{H}$, such that

$$
\begin{aligned}
& i^{2}=j^{2}=k^{2}=-1, \\
& i j=-j i=k, \quad j k=-k j=i, \quad k i=-i k=j,
\end{aligned}
$$

which mutually commute:

$$
[i, @]=[j, @]=[k, @]=0 .
$$

An arbitrary CQ number can be written as

$$
q \equiv @ t+i x+j y+k z
$$

with $t, x, y, z \in \mathbb{C}$. We further introduce a complex conjugate operation * which takes

$$
@ \rightarrow @^{*}=-@
$$

but leaves $i, j$, and $k$ unchanged, as well as a quaternionic conjugate operation -, which leaves @ unchanged but takes

$$
i \rightarrow \bar{i}=-i, \quad j \rightarrow \bar{j}=-j, \quad \text { and } \quad k \rightarrow \bar{k}=-k .
$$

Note that if $o_{1}, o_{2} \in \mathbb{C} \otimes \mathbb{H}$ are two CQ numbers, the order of the product $\mathrm{O}_{1} \mathrm{O}_{2}$ is reversed under quaternionic conjugation only:

$$
\left(o_{1} O_{2}\right)^{*}=o_{1}^{*} O_{2}^{*} \quad \text { but } \quad \overline{o_{1} O_{2}}=\bar{o}_{2} \bar{o}_{1} .
$$

We further define a trace on the complex quaternions via

$$
\operatorname{tr}: \mathbb{C} \otimes \mathbb{H} \rightarrow \mathbb{C}, q \mapsto \frac{1}{2}(q+\bar{q}),
$$

which is $\mathbb{C}$-linear and satisfies

$$
\operatorname{tr}(p q)=\operatorname{tr}(q p)
$$


for $p, q \in \mathbb{C} \otimes \mathbb{H}$.

We label space-time (and other Lorentz contravariant quantities usually denoted by four vectors) by a purely imaginary CQ number

$$
q \equiv @+i x+j y+k z
$$

with $t, x, y, z \in \mathbb{R}$, identify this subspace with Minkowski space, and denote it by $\mathbb{M}$. Complex conjugation * and quaternionic conjugation ${ }^{*}$ then correspond to time reversal $(\mathrm{T})$ and parity $(\mathrm{P})$ transformations, respectively.

The corresponding covariant quantity is given by its quaternionic conjugate or parity reversed CQ number,

$$
\bar{q}=@ t-i x-j y-k z,
$$

yielding the proper time interval

$$
-\bar{q} q=-q \bar{q}=t^{2}-x^{2}-y^{2}-z^{2} .
$$

Defining a scalar product on $\mathbb{M}$

$$
\begin{aligned}
\langle p, q\rangle & \equiv \frac{1}{4}(\overline{(p-q)}(p-q)-\overline{(p+q)}(p+q)) \\
& =-\frac{1}{2}(\bar{p} q+\bar{q} p) \\
& =-\frac{1}{2}(p \bar{q}+q \bar{p}),
\end{aligned}
$$

we find $-\bar{q} q=\langle q, q\rangle$. With $p=@ E+i p_{x}+j p_{y}+k p_{z}$ and $q=@ t+i x+j y+k z$, we have

$$
\langle p, q\rangle=E t-p_{x} x-p_{y} y-p_{z} z
$$

Note that @ $, i, j, k$ form an orthonormal basis of $\mathbb{M}$.

Let $n=i n_{x}+j n_{y}+k n_{z}$ with $n \bar{n}=n_{x}^{2}+n_{y}^{2}+n_{z}^{2}=1$ and $n_{x}, n_{y}, n_{z} \in \mathbb{R}$ be a quaternionic imaginary unit vector. Then a Lorentz transformation is simply given by

$$
q \rightarrow q^{\prime}=\omega q \bar{\omega}^{*}
$$

where

$$
\omega=e^{\frac{1}{2} n \theta}=\cos \frac{\theta}{2}+n \sin \frac{\theta}{2}
$$

for a rotation by an angle $\theta$ around $n$, or

$$
\omega=e^{\frac{1}{2} n @ \Lambda}=\cosh \frac{\Lambda}{2}+@ n \sinh \frac{\Lambda}{2}
$$


for a boost by a Lorentz angle $\Lambda$ in direction $n$.

Clearly the covariant CQ number $\bar{q}$ transforms as

$$
\bar{q} \rightarrow \bar{q}^{\prime}=\omega^{*} \bar{q} \bar{\omega}
$$

With $\omega \bar{\omega}=\bar{\omega} \omega=1$, the Lorentz invariance of the scalar product $\langle p, q\rangle$ is evident.

\section{Dirac equation}

We now apply the complex-quaternionic formulation of special relativity to relativistic wave equations. We begin with the simplest case, i.e., a free scalar field. Let $\Phi(q)$ be a function of one purely imaginary CQ number $q \in \mathbb{M}$, which transforms as a scalar under Lorentz transformations:

$$
\Phi(q) \rightarrow \Phi^{\prime}\left(q^{\prime}\right)=\Phi(q)
$$

The contravariant differentiation operator is defined by

$$
D \equiv @ \frac{\partial}{\partial t}-i \frac{\partial}{\partial x}-j \frac{\partial}{\partial y}-k \frac{\partial}{\partial z}=@ \partial_{t}-i \partial_{x}-j \partial_{y}-k \partial_{z}
$$

and transforms according to

$$
D \rightarrow D^{\prime}=\omega D \bar{\omega}^{*}
$$

Note that $-\bar{D} q=4$, which is equivalent to $\partial_{\mu} x^{\mu}=4$ in standard notation. With the Lorentz invariant operator $-\bar{D} D$ the free Klein-Gordon equation reads

$$
\left(-\bar{D} D+m^{2}\right) \Phi(q)=0
$$

where the real scalar $m$ represents the particle mass. The generally known solutions of (19) may be written as

$$
\Phi(q)=e^{@\langle p, q\rangle},
$$

where the energy-momentum $p=@ E+i p_{x}+j p_{y}+k p_{z}$ satisfies

$$
-\bar{p} p=E^{2}-p_{x}^{2}-p_{y}^{2}-p_{z}^{2}=m^{2} \text {. }
$$


In principle, one could also write down solutions of the form

$$
\Phi(q)=e^{n\langle p, q\rangle},
$$

where $n$ is an arbitrary quaternionic imaginary unit vector. These solutions, however, do not correspond to any physically new states and, in contrast to (201), are not ordinary complex functions. We hence give them no further consideration.

Within this framework, Dirac's equation for the two-component field $\left(\psi_{1}, \psi_{2}\right)^{\mathrm{T}}$ is given by

$$
\left(\begin{array}{cc}
-m & D \\
\bar{D} & -m
\end{array}\right)\left(\begin{array}{l}
\psi_{1} \\
\psi_{2}
\end{array}\right)=0 .
$$

This equation is covariant if $m$ is a real scalar and the component fields transform like

$$
\psi_{1}^{\prime}=\omega \psi_{1} \quad \text { and } \quad \psi_{2}^{\prime}=\omega^{*} \psi_{2} .
$$

This shows that $\psi_{1,2}$ have to be CQ-valued in general. Therefore, Lorentz transformations are represented by diagonal matrices (23), which is clearly a simplification compared to the usual spinor representations used in Dirac's theory [2].

Iteration of (22) yields the Klein-Gordon equation (19) for every component $\psi_{1,2}$ and therefore the relativistic dispersion

$$
E^{2}=\vec{p}^{2}+m^{2},
$$

where $\vec{p}$ is the spatial momentum. From (23) for rotations one finds that, as $\omega(2 \pi)=-1$, particles obeying (22) have spin one-half.

Solving Dirac's equation in the rest frame $(\vec{p}=0)$ we obtain the solutions

$$
\Psi=\left(\begin{array}{c}
\psi \\
\pm \psi
\end{array}\right) e^{\mp @ m t}, \quad \psi \in \mathbb{C} \otimes \mathbb{H},
$$

where the upper sign holds for particles $(E>0)$ and the lower sign for antiparticles $(E<0)$. If one regards $\mathbb{C} \otimes \mathbb{H}$ as a four-dimensional complex vector space, (24) represents eight linearly independent solutions of (22), i.e., twice the number as for the ordinary Dirac equation [2]. This doubling of solutions has led Edmonds [5] and Gough [8] to investigate the possibility of the existence of hidden quantum numbers and associated observables. De 
Leo [7] proposed that one may reduce the number of solutions by transforming (22) into a one-component equation which then requires left and right multiplications by quaternions. We show in Section 6 below that there exists an additional symmetry of (22), which reduces the number of linearly independent solutions from eight to four through gauge invariance.

Solutions with finite velocity are obtained by writing the exponent in (24) as a Lorentz scalar. The Ansatz

$$
\Psi_{ \pm}=\left(\begin{array}{c}
\varphi_{ \pm}(p) \\
\xi_{ \pm}(p)
\end{array}\right) e^{\mp @\langle p, q\rangle}
$$

yields the linear system of equations

$$
\left(\begin{array}{cc}
m & \pm @ p \\
\pm @ \bar{p} & m
\end{array}\right)\left(\begin{array}{c}
\varphi_{ \pm}(p) \\
\xi_{ \pm}(p)
\end{array}\right)=0 .
$$

This implies the spinor condition

$$
\xi_{ \pm}(p)=\mp \frac{1}{m} @ \bar{p} \varphi_{ \pm}(p)
$$

and the energy-momentum relation

$$
p \bar{p}+m^{2}=-E^{2}+\vec{p}^{2}+m^{2}=0 .
$$

Note that (27) is invariant under Lorentz transformations and is satisfied by (24).

\section{Spin and spin directions}

In the previous section we have seen that the solutions of (22) describe particles with spin one-half. For further investigations note that, as the spin operator is the generator of rotations in the rest frame, we have to restrict ourselves to particles in their rest frame, which are represented by states of the form (24). Let us first identify the components of the spin operator. Consider a rotation around the $x$-axis by an angle $\theta$. With $\omega=\omega^{*}$ we obtain

$$
\omega \psi_{1,2}=e^{\frac{1}{2} i \theta} \psi_{1,2}=e^{-@ \frac{\varrho}{2} i \theta} \psi_{1,2} .
$$

The components of the spin operator are now easily read off to be

$$
S_{x} \equiv \frac{@}{2} i, \quad S_{y} \equiv \frac{@}{2} j, \quad \text { and } \quad S_{z} \equiv \frac{@}{2} k \text {. }
$$


With $[i, j]=2 k$ and cyclic permutations one readily verifies that (30) satisfy the angular momentum algebra

$$
\left[S_{x}, S_{y}\right]=@ S_{z}, \quad\left[S_{y}, S_{z}\right]=@ S_{x}, \quad\left[S_{z}, S_{x}\right]=@ S_{y}
$$

For the square of the spin operator we obtain $\vec{S}^{2}=\frac{3}{4}$, as expected. The spin operator $\vec{S}$ is hence implemented through a simple left multiplication with the quaternionic basis elements. Our construction differs from that of De Leo [7] and Gough [9], who proposed operators consisting of simultaneous left and right multiplication with $i, j, k$.

Let us now choose the $z$-axis as spin quantisation direction. The solutions of (22) which are simultaneously eigenstates of $S_{z}$ with eigenvalues $m_{z}= \pm \frac{1}{2}$ are given by

$$
\begin{array}{ll}
m_{z}= & \frac{1}{2}: \quad(1+@ k) \Psi_{0},(i+@ j) \Psi_{0} \\
m_{z}=-\frac{1}{2}: & (@ i+j) \Psi_{0}, \quad(-@-k) \Psi_{0},
\end{array}
$$

where

$$
\Psi_{0}=\left(\begin{array}{l}
1 \\
1
\end{array}\right) e^{-@ m t}
$$

for particles. Antiparticle solutions are constructed similarly. With the usual definition for the raising and lowering operators $S^{ \pm}=S_{1} \pm @ S_{2}$ one easily verifies that the two subspaces

$$
\operatorname{span}_{\mathbb{C}}\left\{(1+@ k) \Psi_{0},(@ i+j) \Psi_{0}\right\} \quad \text { and } \operatorname{span}_{\mathbb{C}}\left\{(i+@ j) \Psi_{0},(-@-k) \Psi_{0}\right\}
$$

are closed under the spin algebra (31). Note that we can convert the spin eigenvectors (32) for the same eigenvalue into each other by right multiplication with quaternionic basis elements, e.g.,

$$
(1+@ k) \Psi_{0} i=(i+@ j) \Psi_{0} \quad \text { and } \quad(@ i+j) \Psi_{0} i=(-@-k) \Psi_{0} .
$$

The right multiplication with the other basis elements $j, k$ does not yield independent states. Hence we can connect the two spin eigenspaces (33) by right multiplication with $i, j, k$. We will return to this issue in Section 6 .

Another peculiarity of the CQ formulation of Dirac's equation is the following. The states

$$
\Psi_{0}, i \Psi_{0}, j \Psi_{0}, k \Psi_{0}
$$


form a basis of the subspace of particle solutions. They are somewhat peculiar, however, as they mix the two spin eigenspaces (33), e.g., we have

$$
\Psi_{0}=\frac{1}{2}(1+@ k) \Psi_{0}+\frac{@}{2}(-@-k) \Psi_{0} .
$$

Note that the eigenvectors (32) of $S_{z}$ satisfy the condition $\Psi^{\mathrm{T}} \bar{\Psi}=0$ (where $\mathrm{T}$ denotes transposition) whereas the states (35) do not. In general, the following two statements are equivalent:

$$
\Psi^{\mathrm{T}} \bar{\Psi}=0 \quad \Longleftrightarrow \quad(\vec{e} \cdot \vec{S}) \Psi= \pm \frac{1}{2} \Psi
$$

where $\vec{e}$ is a unit vector in $\mathbb{R}^{3}$. The dot $\cdot$ denotes the standard scalar product. The right hand side of (37) states that the spin of the particle points in the direction $\vec{e}$. The states (35) are solutions to Dirac's equation and possess spin one-half, but in contrast to the usual situation there is no direction $\vec{e}$ of the spin. Rewriting (37) for the states (24) we obtain the condition

$$
\psi \bar{\psi}=0 \quad \Longleftrightarrow \quad(\vec{e} \cdot \vec{S}) \psi= \pm \frac{1}{2} \psi .
$$

With $\psi=\psi_{0}+i \psi_{1}+j \psi_{2}+k \psi_{3}$, the equation on the left can be rewritten

$$
\psi_{0}^{2}+\psi_{1}^{2}+\psi_{2}^{2}+\psi_{3}^{2}=0
$$

Since $\mathbb{C} \otimes \mathbb{H}$ is isomorphic to the space of complex $2 \times 2$ matrices [10, (39) is equivalent to the statement that $\psi$ is not invertible in $\mathbb{C} \otimes \mathbb{H}$. The condition (38) holds for antiparticles as well.

To prove (37) we start with the eqivalent condition (38). First note that $\vec{e} \cdot \vec{S}=\frac{@}{2} n$ with $n \in \mathbb{H} \backslash \mathbb{R}$. Then multiplying (38) by $\bar{\psi}$ from the right and assuming $\psi \bar{\psi} \neq 0$, we obtain the contradiction

$$
\pm \frac{1}{2}=\frac{@}{2} n \notin \mathbb{R}
$$

Hence, in order to fulfil (38) we need $\psi \bar{\psi}=0$.

The other direction is proven by rewriting $\psi \in \mathbb{C} \otimes \mathbb{H}$ as

$$
\psi=a+@ b+c m+@ d m^{\prime}
$$

with $m, m^{\prime}$ quaternionic imaginary unit vectors and $a, b, c, d \in \mathbb{R}$. Then, by setting $b=0$, we have the condition

$$
\psi \bar{\psi}=(a+c m)(a-c m)-(a+c m) @ d m^{\prime}+@ d m(a-c m)-d^{2}=0,
$$


which yields

$$
a^{2}+c^{2}-d^{2}=0, \quad\left(m m^{\prime}+m^{\prime} m\right) c d=0 .
$$

For $\psi \neq 0$ the equation on the left implies $d \neq 0$. In the first case we have $c=0$ and can choose $n=m^{\prime}$ in (38), in the second case we have $m m^{\prime}+m^{\prime} m=0$ and we can choose $n= \pm \frac{1}{d}(a+c m) m^{\prime}$, which completes the proof.

The above implies that states obeying (37) may be interpreted as ordinary spin one-half particles with usual spin properties. There are, however, two subspaces (331) closed under the spin algebra (31) rather than one, which can be connected by right multiplication with the quaternionic basis elements. In addition to these familiar states there are also states with $\Psi^{\mathrm{T}} \bar{\Psi} \neq 0$ which are not interpretable as ordinary particles. We will return to these peculiarities in Section 6.

\section{Quantum electrodynamics}

So far we considered only free particles. We now turn to Dirac fermions coupled to an external electromagnetic potential, which we describe with the Lagrangian formalism. For free Dirac fermions, the Lorentz invariant Lagrangian density is given by

$$
\begin{aligned}
\mathcal{L}_{0} & =\operatorname{tr}\left(\left(\bar{\psi}_{2}^{*}, \bar{\psi}_{1}^{*}\right)\left(\begin{array}{cc}
-m & D \\
\bar{D} & -m
\end{array}\right)\left(\begin{array}{l}
\psi_{1} \\
\psi_{2}
\end{array}\right)\right) \\
& =\operatorname{tr}\left(\bar{\psi}_{1}^{*} \bar{D} \psi_{1}+\bar{\psi}_{2}^{*} D \psi_{2}-m \bar{\psi}_{1}^{*} \psi_{2}-m \bar{\psi}_{2}^{*} \psi_{1}\right),
\end{aligned}
$$

where the trace is as defined in (5). The free action is given as usual by the integral

$$
\mathcal{S}_{0}=\int_{\mathbb{M}} \mathcal{L}_{0} d^{4} q
$$

where $d^{4} q$ denotes the volume element in $\mathbb{M} \cong \mathbb{R}^{4}$. With $D^{*}=-\bar{D}, \bar{D}^{*}=-D$ we find

$$
\mathcal{L}_{0}^{*}=\operatorname{tr}\left(-\bar{\psi}_{1} D \psi_{1}^{*}-\bar{\psi}_{2} \bar{D} \psi_{2}^{*}-m \bar{\psi}_{1} \psi_{2}^{*}-m \bar{\psi}_{2} \psi_{1}^{*}\right),
$$

which with integration by parts yields the reality condition $\mathcal{S}_{0}^{*}=\mathcal{S}_{0}$. The trace in (42) implies $\overline{\mathcal{S}}_{0}=\mathcal{S}_{0}$ such that $\mathcal{S}_{0} \in \mathbb{R}$. 
To couple the Dirac fields to the electromagnetic potential we require invariance under the local gauge transformation

$$
\Psi \rightarrow \Psi^{\prime}=\Psi e^{-@ e \alpha(q)}
$$

with $e$ a real constant and $\alpha: \mathbb{M} \rightarrow \mathbb{R}$ a scalar function. This implies the minimal coupling procedure $D \rightarrow D+@ e A$ in (22) and the transformation rules for the vector field $A \equiv @ \phi+i A_{x}+j A_{y}+k A_{z}$ :

$$
\begin{aligned}
& A \rightarrow \omega A \bar{\omega}^{*} \quad \text { under Lorentz transformations, } \\
& A \rightarrow A+D \alpha \quad \text { under } U(1) \text { gauge transformations. }
\end{aligned}
$$

The interaction is hence given by the Lagrangian density

$$
\mathcal{L}_{\text {int }}=\operatorname{tr}\left(@ e \bar{\psi}_{1}^{*} \bar{A} \psi_{1}+@ e \bar{\psi}_{2}^{*} A \psi_{2}\right),
$$

which is real as $A^{*}=-\bar{A}$ and $\bar{A}^{*}=-A$. We identify $e$ with the electric charge. The electromagnetic field strength for $A$ is given by [3]

$$
F \equiv \frac{1}{2}(\bar{D} A-\overline{\bar{D} A})
$$

and the Lagrangian density for the free electromagnetic field is

$$
\mathcal{L}_{A}=\frac{1}{4}\left(F^{2}+\left(F^{*}\right)^{2}\right)
$$

which is real since $\bar{F}=-F$. The Lagrangian density of quantum electrodynamics is hence

$$
\mathcal{L}_{\mathrm{QED}}=\mathcal{L}_{0}+\mathcal{L}_{\text {int }}+\mathcal{L}_{A}
$$

From the non-relativistic limit of Dirac's equation, we can further read off the $g$-factor of the electron to equal $g=2$.

\section{Quaternionic gauge invariance and the con- nection to the conventional Dirac equation}

We have seen in Section 3 that a doubling of solutions occurs in the CQ version of Dirac's equation. This is also evident from the existence of two closed spin eigenspaces (33) rather than one. We have seen that these two eigenspaces are connected by right multiplication with $i, j, k$. 
We generalize these transformations to

$$
\Psi \rightarrow \Psi^{\prime}=\Psi e^{-n \beta},
$$

where $n=i n_{x}+j n_{y}+k n_{z}$ is a quaternionic imaginary unit vector and $\beta \in \mathbb{R}$. Note that $e^{-n \beta}$ can be represented in the basis $1, i, j, k$ of $\mathbb{H}$ (see also (13)). The Lagrangian density (42) is, by use of (6), invariant under this transformation. This is, however, not a physical symmetry of the system but rather an invariance of description within the CQ formulation of Dirac's equation, as we will explain now.

Note first that the unit quaternion $e^{-n \beta}$ represents a point on the three sphere $\mathcal{S}^{3} \subset \mathbb{R}^{4}$. Therefore, (52) represents a global $S U(2)$ gauge symmetry. The standard procedure to introduce a gauge symmetry is to require the system to be invariant under a local gauge transformation. To meet this requirement one introduces a gauge field (like $A$ for the $U(1)$ gauge transformations in Section 5) which is minimally coupled to the matter field $\Psi$. This coupling represents the interaction of the matter with the gauge field. The extension of (52) to a local gauge transformation, i.e., $\beta \equiv \beta(q)$, however, does not seem possible within the CQ formulation of Dirac's equation presented here. To see this, consider for simplicity $\beta(q)=x$ and introduce a gauge field $B$ by replacing the differentiation operator $D$ by $D+B$. Then we have to require

$$
((D+B) \Psi)^{\prime} \stackrel{!}{=}(D+B)^{\prime} \Psi^{\prime}=D \Psi^{\prime}+B^{\prime} \Psi^{\prime}
$$

under the (local) transformation (52). For $\beta(q)=x$ this yields

$$
(D \Psi) e^{-n x}+B \Psi e^{-n x}=(D \Psi) e^{-n x}+i \Psi n e^{-n x}+B^{\prime} \Psi e^{-n x},
$$

where we have used the definition of the differentiation operator $D$ (see (17)). As $n \in \mathbb{H}$ and there is no general rule to express $\Psi n$ in terms of $n \Psi$, it is impossible to satisfy (54) with $B$ a CQ-valued function or matrix [11].

It is hence not possible to elevate the global "gauge" symmetry (52) to a true and local gauge symmetry. The symmetry is, however, a gauge symmetry, as it corresponds to an invariance of our description (as gauge symmetries generally do) rather than an invariance of the physical system. It is significant for the interpretation of the CQ formulation of Dirac's equation, as it connects the two spin eigenspaces (33) but leaves the spin eigenvalues invariant. Identifying states connected by quaternionic gauge transformations (152) 
with each other, the number of particle solutions of the free Dirac equation (22) reduces from two to one for each spin eigenvalue. Note that (52) is trivially extended to quantum electrodynamics with $A \rightarrow A$.

Recalling (36), we see that states with $\Psi^{\mathrm{T}} \bar{\Psi} \neq 0$ correspond to superpositions of states formulated in different quaternionic gauges. The crucial point is that no gauge transformation exists which transforms a state with $\Psi^{\mathrm{T}} \bar{\Psi} \neq 0$ into a state which belongs to only one of the subspaces (33), e.g., there is no solution for $n$ and $\beta$ satisfying

$$
\Psi_{0} e^{-n \beta}=a(1+@ k) \Psi_{0}+b(@ i+j) \Psi_{0}, \quad a, b \in \mathbb{C} .
$$

By contrast, spin eigenstates can be transformed by (52) to states which belong to any one of the spin eigenspaces (33). For example,

$$
\frac{1}{\sqrt{2}}(1+@ k) \Psi_{0}+\frac{1}{\sqrt{2}}(i+@ j) \Psi_{0}=(1+@ k) \Psi_{0} e^{i \frac{\pi}{4}} .
$$

This does, however, not provide us with a definite answer to the question whether states with $\Psi^{\mathrm{T}} \bar{\Psi} \neq 0$ should be interpreted physically or should be excluded from the theory by requiring $\Psi^{\mathrm{T}} \bar{\Psi}=0$ for physical states explicitly. The contemplations summarized above suggest the latter.

To verify that there are no further gauge symmetries, note that the Lagrangian density (42) is invariant under right multiplication of a CQ number $\Sigma$ only if $\Sigma \bar{\Sigma}^{*}=1$. Then the statement

$$
\Sigma \bar{\Sigma}^{*}=1 \Rightarrow \Sigma=c q,
$$

where $c \in \mathbb{C}, q \in \mathbb{H}$, and $|c||q|=1$, shows that there are no gauge symmetries besides the $U(1)$ symmetry as described in Section 5 and the quaternionic gauge transformations (52). The proof of (57) is straightforward, but somewhat to technical for this article.

We conclude this section by relating the CQ version of Dirac's equation (22) to the standard formulation with complex matrices [2]. For this purpose, we expand the CQ spinors $\psi_{1,2}$ in up- and down-spin components, where we can restrict ourselves due to (52) (at least for states satisfying $\Psi^{\mathrm{T}} \bar{\Psi}=0$ ) to the first of the two subspaces (33), i.e., we write

$$
\psi_{1}=(1+@ k) c_{1}+(@ i+j) c_{2}, \quad \psi_{2}=(1+@ k) c_{3}+(@ i+j) c_{4}
$$

with $c_{i}: \mathbb{M} \rightarrow \mathbb{C}$. Inserting this in (22) yields four independent equations which combine to

$$
\left(@ \gamma^{\mu} \partial_{\mu}-m\right) \mathcal{C}=0 \text { with } \mathcal{C}=\left(c_{1}, c_{2}, c_{3}, c_{4}\right)^{\mathrm{T}},
$$


where the gamma matrices are given in the chiral representation:

$$
\gamma^{0}=\left(\begin{array}{ll}
\mathbf{0} & \mathbf{1} \\
\mathbf{1} & \mathbf{0}
\end{array}\right), \quad \gamma^{i}=\left(\begin{array}{cc}
\mathbf{0} & \sigma^{i} \\
-\sigma^{i} & \mathbf{0}
\end{array}\right)
$$

with $\mathbf{0}$ and $\mathbf{1}$ are the $2 \times 2$ zero and unit matrix and $\sigma^{i}$ the Pauli matrices. The CQ formulation with $\Psi^{\mathrm{T}} \bar{\Psi}=0$ presented here is hence equivalent to the standard complex formulation of Dirac's equation.

\section{Conclusion}

In this article, we have extended the complex-quaternionic formulation of special relativity [3] to Dirac's equation, obtaining an equation for a twocomponent CQ field. In this formulation there is no need to invoke Dirac matrices, and Lorentz transformations are given by diagonal matrices rather than complicated spinor representations. The price to pay for these simplifications is, however, outrageous. First, we encountered a doubling of solutions, a problem which we overcame by attributing them to different gauge choices of a global $S U(2)$ gauge symmetry. Second, we encountered particle solutions with spin one-half but without a spin quantisation direction. According to conventional quantum mechanics these solutions appear to be unphysical, but this did not emerge from the formalism we developed above. This led us to derive a condition to ban them from the Hilbert space, which we imposed a posteriori. Furthermore, there are mathematical problems inherent to the formulation due to the fact that $\mathbb{C} \otimes \mathbb{H}$ is not a division algebra [4, 12.

In summary, we found no indication that the CQ formulation of Dirac's equation would lead to a deepening of our understanding of relativistic fermions. In our opinion, the problems we encountered with this formulation eradicate the gain in formal elegance we were hoping to obtain.

\section{Acknowledgment}

One of us (DS) was supported by the German Research Foundation (DFG) through GK 284. 


\section{References}

[1] Dirac P A M 1928 Proc. R. Soc. A117 610

Dirac P A M 1928 Proc. R. Soc. A118 351

[2] Bjorken J D and Drell S D 1964 Relativistic quantum mechanics (New York: McGraw-Hill)

[3] Greiter M and Schuricht D 2003 Eur. J. Phys. 24397

[4] Adler S 1995 Quaternionic Quantum Mechanics and Quantum Fields (Oxford: Oxford University Press)

[5] Edmonds J D 1973 Found. Phys. 3313

Edmonds J D 1976 Found. Phys. 633

[6] Rotelli P 1989 Mod. Phys. Lett. A4 933

[7] De Leo S 1996 Int. J. Mod. Phys. A11 3973

[8] Gough W 1987 Eur. J. Phys. 8164

[9] Gough W 1986 Eur. J. Phys. 735

[10] Waerden B L van der 1985 A History of Algebra (Berlin: Springer)

[11] In different frameworks of the quaternionic formulation of Dirac's equation one can construct local $S U(2)$ gauge theories by introducing gauge fields including left and right multiplication with quaternions (see: Morita K 1982 Progr. Theor. Phys. 67 1860; De Leo S 1996 J. Phys. G: Nucl. Part. Phys. 22 1137).

[12] Dixon G M 1994 Division Algebras: Octonions, Quaternions, Complex Numbers and the Algebraic Design of Physics (Dordrecht: Kluwer Academic Publishers) 